\title{
LAS COMUNIDADES DE PALMERAS EN LOS ECOSISTEMAS FORESTALES INUNDABLES DE LA AMAZONIA PERUANA
}

Kahn Francis ( $\left.{ }^{*}\right)$

M ejía Kember $\left(^{* *}\right)$

\section{RESUMEN}

Se realizaron estudios de palmeras en los ecosistemas forestales inundables más representativos en el bajo U cayali (Ilanura amazónica) yen el al to Huallaga (piedemonte oriental andino). Estos ecosistemas se caracterizan por la composición específica, densidad y distribución vertical de la comunidad de palmeras. Las palmeras pueden formar poblaciones casi monoespecificas, como en los pantanos de Mauritia flexuosa (aguajales); 6 en comunidades multiespecfficas, como en los bosques de quebrada, en los valles de altura, en donde predominan Euterpe precatoria Jessenia bataua, M auritia flexuosa y Socratea exorrhiza y en los bosques de suelos aluviales, periódicamente inundados (restinga), dominados por algunas especies de los géneros Astrocaryum. Phitelephas y Scheelea. En los bosques que son periódicamente inundados por aguas negras (tahuampa), la diversidad de palmeras es baja; sin embargo, la densidad es frecuentemente alta.

\section{INTRODUCCION}

Los ecosistemas forestales inundables cubren vastas áreas en la amazonía peruana (López Parodi y Freitas 1990; ONIERN, 1975; Salo et al, 1986). En los valles de las tierras bajas de los principales ríos, están diversificados y constituyen un mosaico vegetacional complejo (Encarnación, 1985); mientras que en los valles de las tierras altas, son mas bien homogénos y difieren claramente de los bosques de altura por su composición florística, fisonomía y estructura (Granville, 1976; Kahn \& Castro, 1985; Oldeman, 1974).

Las palmeras se encuentran en todos los bosques de la cuenca amazónica; las formas arborescentes, grandes, son frecuentes en los suelos inundados o hidromórficos, en donde usualmente forman poblaciones densas. Algunas de estas especies están claramente relacionadas con la dinámica fluvial y con las propiedades del agua (carga de sedimentos, acidez), como fue reportado por Spruce (1871) 
Las comunidades de palmeras de los ecosistemas inundables más representativos son descritas en dos regiones de la amazonía peruana; una localizada en la llanura amazónica y la otra en el piedemonte oriental andino. Para cada uno de los bosques estudiados se especifica la composición florística, riqueza específica, densidad y distribución vertical de las palmeras. La comparación de esta información pone de manifiesto las características de cada comunidad de palmeras como elementos para la definición e identificación de los ecosistemas de los bosques inundables.

\section{METODOS}

\section{$2.1 \quad$ Areas de estudio}

En el valle del bajo U cayali, cerca de J enaro Herrera (40 55'S; $73040^{\prime}$ 0), los ecosistemas inundables más representativos son conocidos localmente como "tahuampa', "restinga", "aguajal" y "bosque de quebrada" (Encarnación, 1985). Los bosques de tahuampa son inundados periódicamente por aguas negras; este ecosistema, raramente cubre grandes áreas, y no está claramente determinado como el igapó brasileño (A dis, 1984; Prance, 1979, Takeuchi, 1962). Los bosques de restinga son la última fase de la sucesión primaria, la que comienza en el herbazal, sobre los sedimentos depositados por el río U cayali (agua blanca); esta vegetación corresponde al concepto brasileño de bosque de várzea (Prance, 1979). Los bosques de aguajal son palmerales en depresiones permanentemente inundadas, paralelos al lecho del río yen los que el substrato arcilloso retiene la lluvia y el agua del río entra a estas depresiones sólo en tiempo de altas inundaciones; el suelo de los aguajales (histosol) está compuesto por una acumulación de materia orgánica, ligeramente descompuesta en aguas ácidas ( $\mathrm{pH}=3.5$ ). Los bosques de quebrada se encuentran en suelos hidromórficos (gleysoles), que están irregularmente inundados durante la estación Iluviosa. En el al to Huallaga, cerca de U chiza (80 17'S) (760 26'W), la vegetación de tierras bajas está dominada por bosques de quebrada, irregularmente inundados durante la estación lluviosa, y por bosques sobre suelos al uviales anualmente inundados por el río Huallaga.

\section{$2.2 \quad$ Estudio de las palmeras}

En el bajo U cayali, se inventarió las comunidades de palmeras: Se coleccionó para ello una parcela de $0.4 \mathrm{Ha}$. en un bosque sobre suelos aluviales periódicamente inundados, y dos parcelas de $1 \mathrm{Ha}$ : una en un pantano de $\mathrm{M}$. flexuosa y en otra un bosque de quebrada, respectivamente. La comunidad de palmeras en bosques periódicamente inundados por aguas negras sólo fue descrita cual itativamente, debido a su baja diversidad específica. 
En el al to Huallaga, se estudió las palmeras en dos parcelas dc $0.8 \mathrm{Ha}$ : en un bosque de quebrada y en una parcela de $0.4 \mathrm{Ha}$, en un bosque en suelos aluviales periódicamente inundados. El conteo incluyó todas las palmeras, excepto en las dos parcelas de $1 \mathrm{Ha}$ en el bajo $\mathrm{U}$ cayali, en donde no se consideraron los individuos de menos de $1 \mathrm{~m}$. Para las especies multicaules, cada eje fue contado como una palmera.

\subsection{Identificación y colección de palmeras.}

Se colectaron muestras botánicas de referencia de todas las especies de palmeras encontradas. A Igunas de ellas no pudieron ser identificadas, debido a la falta de muestras comparativas en los herbarios (BH, K, NY, USM, AMAZ): Bactris sp. 1, B. sp.2 y Sheelea sp., o debido ala falta de material fértil: Desmoncus sp. Algunas especies bien conocidas fueron encontradas sin órganos reproductivos en las parcelas estudiadas en el bajo U cayali: Geonoma leptospadix piscicauda G. spixiana Lepidocarvum tessmannii $\overline{\text { Orbignya }}$ polysticha Socratea exorrhiza y Wettinia augusta sin embargo, se recogió material fértil en la misma localidad.

\section{RESULTADOS}

\subsection{Palmeras en bosques inundables en el balo U cayali}

En el bosque de suelos aluviales (restinga), se encontraron once especies en una parcela de $0.4 \mathrm{Ha}$ (Cuadro $\mathrm{N} \cong 1$ ); de ellas, cinco representan el $98.9 \%$ de la comunidad: Astrocaryum macrocalyx Phytelephas macrocarpa Scheelea brachyclada Bactris bifida y Geonoma acaulis. Sobre los $10 \mathrm{~m}$. de altura la densidad de palmeras es muy baja $(0.3 \%)$ y muy alta entre 1-10 m. (353 palmeras / $0.1 \mathrm{Ha}, 50.2 \%$ de la comunidad). A. macrocalyx yL macrocarpa especies mono y multicaules, respectivamente, dominan el estrato de $1-10 \mathrm{~m}$.; ambas especies raramente alcanzan más de $10 \mathrm{~m}$. de altura. La densidad de palmeras menores a 1 metro de altura es al ta ( $49.5 \%$ de la comunidad), a pesar de la inundación prolongada de 3 a 4 meses al año.

En los bosques permanentemente inundados (aguajales), se encontró 11 especies en $1 \mathrm{Ha}$. de estudio (Cuadro $\mathrm{N}$ o 2). Entre ellas, 4 especies representan el $90.2 \%$ de la comunidad de palmeras (sobre $1 \mathrm{~m}$. de altura); Mauritia Fleuxosa, Geonoma acaulis Oenocarpus mapora y Euterpe precatoria: el dosel de estos bosques empantanados está esencialmente compuesto por $M$ auritia flexuosa. Ladensidad de las palmeras sobre los $10 \mathrm{~m}$. es alta, con 207 palmeras por hectárea. El área basal de las palmeras representa el 55\% del área basal total $(31.11 \mathrm{~m} 2 / \mathrm{Ha}$ ), con 336 troncos, incluyendo $230 \mathrm{M}$. flexuosa (138 adultos y 92 troncos jóvenes). 


\section{CUADRO N 1}

RIQUEZA ESPECIFICA, DENSIDAD Y DISTRIBUCION VERTICAL DE LA COMUNIDAD DE PALMERAS EN $0.4 \mathrm{Ha}$ EN BOSQUES PERIODICAMENTE INUNDADOS SOBRE SUELOS ALUVIALES EN EL BAJO UCAYALL

\begin{tabular}{|l|rrrrr|}
\hline \multirow{2}{*}{ ESPECIES } & \multicolumn{5}{c|}{ NUMERO DE INDIVIDUOS } \\
\cline { 2 - 6 } & $0-1 \mathrm{~m}$ & $1-10 \mathrm{~m}>10 \mathrm{~m}$ & TOTAL & $(\%)$ \\
\hline Astrocaryum macrocalyx & 722 & 328 & 0 & 1050 & $(37.33)$ \\
Phytelephas macrocarpa & 25 & 841 & 0 & 866 & $(30.79)$ \\
Scheelea brachyclada & 372 & 97 & 7 & 476 & $(16.92)$ \\
Bactris bifida & 183 & 95 & 0 & 278 & $(9.88)$ \\
Geonoma acaulis & 78 & 34 & 0 & 112 & $(3.98)$ \\
Bactris monticola & 7 & 5 & 0 & 12 & $(0.43)$ \\
Euterpe precatoria & 6 & 2 & 0 & 8 & $(0.28)$ \\
Socratea exorrhiza & 0 & 6 & 0 & 6 & $(0.21)$ \\
Oenocarpus mapora & 0 & 2 & 0 & 2 & $(0.07)$ \\
Bactris maraja & 0 & 1 & 0 & 1 & $(0.03)$ \\
Desmoncus polyacanthos & 0 & 1 & 0 & 1 & $(0.03)$ \\
T OT A L & 1393 & 1412 & 7 & 2812 & \\
\hline
\end{tabular}

\section{CUADRO N²}

RIQUEZA ESPECIFICA, DENSIDAD Y DISTRIBUCION VERTICAL DE LA COMUNIDAD DE PALMERAS EN

$1 \mathrm{Ha}$ DE BOSQUE PERMANENTEMENTE INUNDADO SOBRE HISTOSOL EN EL BANO UCAYAU

\begin{tabular}{|c|c|c|c|c|c|}
\hline \multirow{2}{*}{ ESPECIES } & \multicolumn{5}{|c|}{ NUMERO DE INDIVIDUOS } \\
\hline & $0-1 \mathrm{~m}$ & $1-10 \mathrm{~m}$ & $>10 \mathrm{~m}$ & TOTAL & $(\%)$ \\
\hline Mauritia flexuosa & $\ldots$ & 478 & 167 & 645 & (54.47) \\
\hline Geonoma acaulis & $\cdots$ & 252 & 0 & 252 & (21.28) \\
\hline Denocarpus mapora & $\cdots$ & 119 & 2 & 121 & $(10.21)$ \\
\hline Euterpe precatoria & $\ldots$ & 16 & 34 & 50 & $(4.22)$ \\
\hline Bactris maraja & $\cdots$ & 42 & 0 & 42 & $(3.54)$ \\
\hline Bactris monticola & $\cdots$ & 26 & 0 & 26 & (2.19) \\
\hline Desmoncus polyacanthos & $\cdots$ & 20 & 0 & 20 & (1.68) \\
\hline Bactris bifida & $\cdots$ & 15 & 0 & 15 & (1.26) \\
\hline Socratea exorrhiza & $\cdots$ & 3 & 4 & 7 & $(0.59)$ \\
\hline Astrocaryum macrocalyx & $\ldots$ & 5 & 0 & 5 & $(0.42)$ \\
\hline Scheelea brachyclada & $\cdots$ & 1 & 0 & 1 & $(0.08)$ \\
\hline TOT A L & $\ldots$ & 977 & 207 & 1184 & \\
\hline
\end{tabular}




\section{CUADRON 23}

RIQUEZA ESPECIFICA, DENSIDAD Y DISTRIBUCION VERTICAL DE LA COMUNIDAD DE PALMERAS EN $1 \mathrm{Ha}$ DE BOSQUE DE QUEBRADA EN LOS VALLES DE ALTURAS DEL BAJO UCAYALI

\begin{tabular}{|c|c|c|c|c|c|}
\hline \multirow{2}{*}{ ESPECIES } & \multicolumn{5}{|c|}{ NUMERO DE INDIVIDUOS } \\
\hline & $0-1 \mathrm{~m}$ & $1-10 \mathrm{~m}$ & $>10 \mathrm{~m}$ & TOTAL & (\%) \\
\hline Bactris monticola & $\cdots$ & 866 & 0 & 866 & $(36.38)$ \\
\hline \essenia bataua & $\cdots$ & 313 & 48 & 361 & (15.16) \\
\hline Mauritia flexuosa & $\cdots$ & 218 & 32 & 250 & $(10.50)$ \\
\hline Euterpe precatoria & $\cdots$ & 205 & 45 & 250 & $(10.50)$ \\
\hline Geonoma acaulis & $\cdots$ & 237 & 0 & 237 & (9.95) \\
\hline Socratea exorrhiza & $\cdots$ & 113 & 46 & 159 & $(6.68)$ \\
\hline Lepidocaryum tessmannii (1) & $\cdots$ & 151 & 0 & 151 & $(6.34)$ \\
\hline Oenocarpus mapora & ... & 39 & 1 & 40 & (1.68) \\
\hline Bactris simplicifrons & $\cdots$ & 18 & 0 & 18 & $(0.75)$ \\
\hline Astrocaryum macrocalyx & $\cdots$ & 13 & 0 & 13 & $(0.54)$ \\
\hline Geonoma oligoclona & $\cdots$ & 10 & 0 & 10 & $(0.42)$ \\
\hline Orbignya polysticha (1) & $\cdots$ & 9 & 0 & 9 & $(0.37)$ \\
\hline Bactris sp. 1 (1) & $\cdots$ & 5 & 0 & 5 & $(0.21)$ \\
\hline Geonoma spixiana (1) & ... & 4 & 0 & 4 & (0.16) \\
\hline Bactris acanthocarpoides (1) & $\cdots$ & 3 & 0 & 3 & $(0.12)$ \\
\hline Wettinia augusta & $\cdots$ & 2 & 0 & 2 & $(0.08)$ \\
\hline Geonoma leptospadix (1) & $\cdots$ & 1 & 0 & 1 & $(0.04)$ \\
\hline Geonoma piscicauda (1) & $\cdots$ & 1 & 0 & 1 & $(0.04)$ \\
\hline TOT AL & $\cdots$ & 2208 & 172 & 2380 & \\
\hline
\end{tabular}

(1) Estas espedes, comunes en bosques de altura, se presentan en una isla de suelos bien drenados de $+/-500 \mathrm{~m}^{2}$.

L a densidad de las palmeras en el sotobosque ( 1 - $10 \mathrm{~m}$.) es de 98 palmeras/ 0.1 $\mathrm{Ha}$; la mitad de ellas son juveniles acaules y con tronco de $M$. flexuosa. La densidad de Astrocaryum macrocalyx y Scheelea brachyclada es muy baja, a pesar que ambas son especies dominantes en las dos restingas' que limitan la depresión empantanada de $M$. flexuosa: sin embargo, Phytelephas macrocarpa también abundante en las 'restingas" cercanas, está ausente en la depresión.

En el bosque de quebrada se encontró 18 especies en $1 \mathrm{Ha}$. estudiada (Cuadro $\mathrm{N}$ o 3). Entre ellas, 7 fueron localizadas en un área de más 0 menos $500 \mathrm{~m}^{2}$ de suelos más secos dentro de la parcela estudiada. Estas especies, que son muy comunes en las terrazas no inundables (Kahn \& M ejía, 1990), no pueden considerarse como representativas de esta comunidad. Seis especies representan el $89.2 \%$ de la comunidad de palmeras (sobre $1 \mathrm{~m}$. de altura); Bactris monticula lessenia bataua Euterpe precatoria 
M auritia flexuosa Geonoma acaulis, Socratea exorrhiza. El área basal de las palmeras representa el $36.3 \%$ del área basal total $\left(26.34 \mathrm{~m} .{ }^{2} / \mathrm{Ha}\right.$. $)$, con 279 troncos, de los cuales 172 alcanzan mas de $110 \mathrm{~m}$. de altura. Sus follajes constituyen el mayor componente de la copa del bosque. B. montícola es la especie dominante en el sotobosque de los suelos hidromórficos, mientras que Lepidocaryurn tessmannii alcanza una muy al ta densidad en la pequeña isla de suelos más secos.

En los bosques periódicamente inundados por aguas negras (tahuampa), la riqueza de palmeras es muy baja. L as especies más comunes son A strocaryum jauari una palmera arborescente multicaule, que alcanza hasta $20 \mathrm{~m}$. de altura; Bactris riparia monocaule; Bactris concinna y: a maraja multicaules; son palmeras pequeñascon menos de $10 \mathrm{~m}$. de altura. Estas especies cespitosas constituyen matas densas en áreas pequeñas.

\subsection{Palmeras en bosques inundables en el alto Huallaga.}

En los bosques de quebrada, las 15 especies encontradas estuvieron presentes en cada una de las parcelas de $0.8 \mathrm{Ha}$. estudiadas (Cuadro N 2 4), la densidad por cada especies acumulada en $1.6 \mathrm{Ha}$.

Nueve de ellas representan el $93.7 \%$ de la comunidad; Geonoma brongniartii Jessenia bataua, Euterpe precatoria Geonoma macrostachys Oenocarpus mapora Socratea exorrhiza, Iriartea del toidea, Wettinia augusta y M auritia flexuosa. La densidad de las palmeras sobre los $10 \mathrm{~m}$. de altura es baja (1.6\% de la comunidad), con 43 troncos, de los cuales 22 pertenecen a M. flexuosa. M uchas palmeras han sido cortadas, y muchos troncos descompuestos del $\_$. bataua y de $\mathrm{M}$. flexuosa yacen en el piso del bosque.

En los bosques en suelos aluviales periódicamente inundados, se encontraron 15 especies en $0.4 \mathrm{Ha}$. estudiadas (Cuadro $\mathrm{N} 0$ 5). Entre ellas, cuatro constituyen el $86.0 \%$ de la comunidad: A strocaryum murumuru Euterpe precatoria Oenocarpus mapora y Geonoma macrostachys. A. murumuru es la especie dominante en el sotobosque; la densidad de palmeras sobre los $10 \mathrm{~m}$. de altura es baja ( $0.7 \%$ de la comunidad).

\section{DISCUSION}

Se encontró una alta similaridad florística en los ecosistemas estudiados, indicada por la presencia de las mismas especies en los diversos inventarios.

En el bajo U cayali, las 6 especies que son comunes a los tres inventarios representan el $26.6 \%, 38.9 \%$ y $65.8 \%$ de las comunidades de palmeras (sobre $1 \mathrm{~m}$. de altura), en los bosques de suelos aluviales, periódicamente inundados, en el pantano de 


\section{CUADRO N4}

RIQUEZA ESPECIFICA, DENSIDAD Y DISTRIBUCION VERTICAL DE LA COMUNIDAD DE PALMERAS EN $1.6 \mathrm{Ha}$ DE BOSQUE DE QUEBRADA EN EL ALTO HUALLAGA

\begin{tabular}{|l|rrrrr|}
\hline \multirow{2}{*}{ ESPECIES } & \multicolumn{5}{c|}{ NUMERO DE INDIVIDUOS } \\
\cline { 2 - 6 } & $0-1 \mathrm{~m}$ & $1-10 \mathrm{~m}>10 \mathrm{~m}$ & TOTAL & $(\%)$ \\
\hline Geonoma brongriartii & 103 & 320 & 0 & 423 & $(15.40)$ \\
lessenia bataua & 231 & 160 & 7 & 398 & $(14.49)$ \\
Euterpe precatoria & 239 & 88 & 1 & 328 & $(11.94)$ \\
Geonoma macrostachys & 152 & 147 & 0 & 299 & $(10.89)$ \\
Oenocarpus mapora & 109 & 184 & 0 & 293 & $(10.67)$ \\
Socratea exorrhiza & 206 & 57 & 9 & 272 & $(9.90)$ \\
Iriartea deltoidea & 153 & 104 & 0 & 257 & $(9.36)$ \\
Wettinia augusta & 127 & 42 & 4 & 173 & $(6.30)$ \\
Mauritia flexuosa & 22 & 85 & 22 & 129 & $(4.69)$ \\
Bactris sp. & 32 & 50 & 0 & 82 & $(2.98)$ \\
Geonoma acaulis & 10 & 23 & 0 & 33 & $(1.20)$ \\
Astrocaryum macrocalyx & 10 & 22 & 0 & 32 & $(1.16)$ \\
Bactris maraja & 2 & 15 & 0 & 17 & $(0.61)$ \\
Desmoncus sp. & 0 & 7 & 0 & 7 & $(0.25)$ \\
Chamaedorea cf. lanceolata & 0 & 2 & 0 & 2 & $(0.07)$ \\
\cline { 2 - 6 } TO T A L & 1396 & 1306 & 43 & 2745 & \\
\hline
\end{tabular}

\section{CUADRO N 25}

RIQUEZA ESPECIFICA, DENSIDAD Y DISTRIBUCION VERTICAL DE LA COMUNIDAD DE PALMERAS EN 0.4 Ha DE BOSQUE PERIODICAMENTE INUNDADO SOBRE SUELOS ALUVIALES EN EL ALTO HUALLAGA

\begin{tabular}{|l|rrrrr|}
\hline \multirow{2}{*}{ ESPECIES } & \multicolumn{5}{c|}{ NUMERO DE INDIVIDUOS } \\
\cline { 2 - 6 } & $0-1 \mathrm{~m}$ & $1-10 \mathrm{~m}>10 \mathrm{~m}$ & TOTAL & $(\%)$ \\
\hline Astrocaryum macrocalyx & 230 & 352 & 0 & 582 & $(49.53)$ \\
Euterpe precatoria & 176 & 67 & 1 & 244 & $(20.76)$ \\
Oenocarpus mapora & 37 & 93 & 0 & 130 & $(11.56)$ \\
Geonoma macrostachys & 35 & 19 & 0 & 54 & $(4.59)$ \\
Phytelephas macrocarpa & 2 & 34 & 0 & 36 & $(3.06)$ \\
Socratea exorrhiza & 16 & 16 & 3 & 35 & $(2.97)$ \\
Scheelea sp. & 9 & 11 & 0 & 20 & $(1.70)$ \\
Chelyocarpus ulei & 8 & 10 & 0 & 18 & $(1.53)$ \\
Bactris sp. 2 & 4 & 12 & 0 & 16 & $(1.36)$ \\
Chamaedorea cf. lanceolata & 5 & 7 & 0 & 12 & $(1.02)$ \\
Iriartea deltoidea & 0 & 8 & 0 & 8 & $(0.68)$ \\
Geonoma acaulis & 3 & 4 & 0 & 7 & $(0.59)$ \\
Mauritia flexuosa & 1 & 2 & 3 & 6 & $(0.51)$ \\
lessenia bataua & 1 & 2 & 1 & 4 & $(0.34)$ \\
Geonoma maxima & 1 & 2 & 0 & 3 & $(0.25)$ \\
TO T A L & 528 & 639 & 8 & 1175 & \\
\hline
\end{tabular}




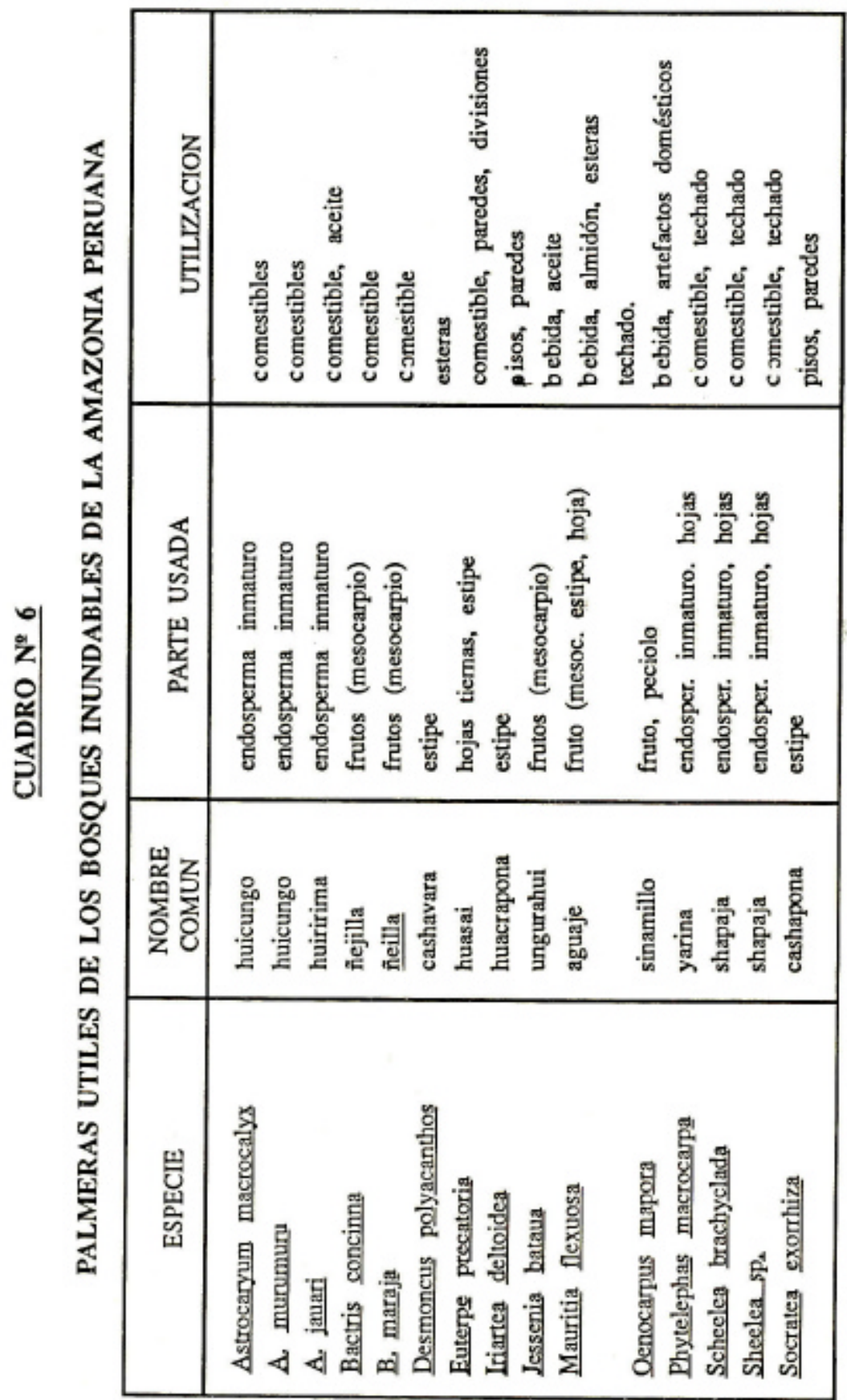


Estos dos últimos bosques tienen en común siete especies, las cuales constituyen el $93.4 \%$ y $76.3 \%$ de su comunidad de palmeras, respectivamente. En el alto Huallaga, 11 de las 18 especies son comunes a ambos ecosistemas y representan el $77.4 \%$ y $93.4 \%$ de las comunidades en los bosques dc quebrada y en el bosque de suelos aluviales, periódicamente inundados, respectivamente. Las características de las comunidades de palmeras en cada uno de los ecosistemas forestales inundables son, sin embargo, muy distintas: Los bosques pantanosos en depresiones permanentemente inundadas están compuestos principalmente de M auritia flexuosa, la cual alcanza muy altas densidades (Cuadro № 2); (Gonzáles Rivadeneyra, 1971; Salazar y Roessl, 1977). Plantas jóvenes y adultas dominan el sotobosque. El aguajal, que es el nombre local de estos bosques pantanosos se deriva de "aguaje', el nombre local de las palmeras. La cercana relación de $M$. flexuosa con el agua es sugerida por los comentarios de Spruce (1871, p. 78): "La opinión prevaleciente o más bien supersticiosa, a lo largo de la Amazonía y Guyana, es que la Mauritia tiene el poder de atraer el agua hacia ella, dondequiera que sea plantada. Los bosques de quebrada en las terrazas no inundables son dominados por cuatro especies: Euterpe precatoria lessenia bataua, Mauritia flexuosa y Socratea exrorrhiza, las que, juntas, constituyen un importante componente del dosel. L bataua es una palmera muy característica de los bosques de quebradas sobre suelos hidromórficos; esta especie fue raramente encontrada en otros ecosistemas inundables. Otras tres especies son particularmente comunes en este ecosistema del piedemonte oriental andino: Iriartea deltoidea Oeonocarpus mapora y W ettinia augusta. La densidad de los troncos de las palmeras de las tres especies arborescentes, E. precatoria J. bataua y $M$. flexuosa es particularmente baja. L as tres proveen productos útiles, tales como aceite, fruta comestible y materiales de construcción (Kahn, 1988; M ejía 1988), (Cuadro №6). Ellas han sido extensivamente cortadas por los nuevos y numerosos habitantes que se han establecido en este valle, atraídos por prospectos de trabajo de una plantación de palmera africana de 4,500 $\mathrm{Ha}$. Los bosques sobre suelos aluviales periódicamente inundados (restinga baja), se caracterizan por la asociación de varias especies de tres géneros: Astro-caryum ( $\&$ macrocalyx y A. murumuru. dos especies cercanamente relacionadas, Ilamadas localmente "huicungo") y Phytelephas (P macrocarpa "yarina", y Scheelea (diversas especies, todas localmente llamadas "shapaja'). En contraste con otros bosques inundables, las palmeras arborescentes no son dominantes en la copa de los bosques de restinga. Por otro lado, las palmeras son el mayor componente del sotobosque, debido a la abundancia de Astrocaryum spp. Y Phytelephas macrocarpa. Poblaciones densas de las últimas son conocidas como "yarinal" y se cree usualmente que guardan relaciones con suelos de buena calidad. La asociación de estos tres géneros de palmeras también ocurre en los bosques ecuatorianos sobre suelos aluviales (Balslev et al., 1987). Los bosques que son periódicamente inundados por aguas negras (tahuampa), se caracterizan por la presencia de palmeras ripícolas, tales como: Astrocaryum jauari y Bactris maraja cuyos frutos son diseminados por los peces (Gottsberger, 1978; Goulding, 1980). Estas especies también se presentan en las orillas de la mayoría de los ríos amazónicos. Desmoncus polyacanthos una palmera lianescente, 
es comunmente encontrada en los bosques de tahuampa, así como en los suelos inundables, con vegetación baja y en bosques abiertos. La diversidad de las palmeras en cada uno de los bosques inundables en el bajo U cayali, no es severamente diferente: 11 especies, 9 géneros en $0.4 \mathrm{Ha}$, en suelos aluviales periódicamente inundados; 11 especies, 9 géneros en $0.1 \mathrm{H}$ a. en un pantano de $M$. flexuosa; 18 especies, 11 géneros en $1 \mathrm{Ha}$. de bosque de quebrada (en este caso, 2 géneros y 7 especies, que están restringidas a una pequeña área de terreno más seco, deben de ser excluidas). La diversidad de las palmeras es ligeramente más al ta en los bosques de quebrada en el alto Huallaga; 15 especies, 13 géneros en $0.4 \mathrm{Ha}$. En terrenos aluviales periódicamente inundados; 15 especies, 12 géneros, en cada uno de los dos sectores de $0.8 \mathrm{Ha}$. estudiadas de bosques de quebrada. La diversidad de las palmeras en los bosques de altura es claramente más grande: 29 especies, 16 géneros en $0.71 \mathrm{Ha}$ y 34 especies, 21 géneros en $0.5 \mathrm{Ha}$ fueron inventariados ( $\mathrm{K}$ ahn \& M ejía, 1991). En la amazonia central (bajo río N egro), la riqueza de palmeras es también menor en los bosques de quebrada que en los de altura ( $K$ ahn \& C astro, 1985). En la amazonia occidental, la diferencia de riqueza específica entre los ecosistemas forestales de altura y los de depresiones, no es tan notable por la muy baja diversidad de palmeras en esta región de la cuenca (Kahn et al., 1988). La mayoría de las palmeras de suelos inundables que son muy comunes en la amazonia peruana están a lo largo de la cuenca amazónica: Astrocaryum jauari. A. murumuru Bactris concinna B. maraja B. monticola, Desmoncus polvacanthos M auritia flexuosa y Socratea exorrhiza quees frecuente, ya sea en suelos inundados 0 hidromórficos, como en suelos bien drenados ( $\mathrm{K}$ ahn \& Castro, 1985). Otras especies no son encontradas en la región oriental, pero son frecuentes en la amazonia central y la occidental: Astrocaryum macrocalyx Euterpe precatoria Geonoma acaulis Jessenia bataua subsp. bataua J. bataua subsp. Oligocarpa se presenta en las Guyanas). Las especies occidentales son: O enocarpus mapora, Phtelephas Scheelea spp., y Geonoma brongniartii G. macrostachys Iriartea deltoidea y Wettinia augusta. Las cuales son particularmente frecuentes en el piedemonte oriental andino.

\section{BIBLIOGRAFIA}

ADIS, J., 1984 Seasonal igapó -forest of central A mazonianblackwaterriversandtheir terrestrial arthropod fauna. In: H. Sioli (Editor), The A mazon Limnology and landscape ecology of a mighty tropical river and its basin. Dr. Junk Publisher. Dordrecht, Boston, Landcaster, p. 245 - 268

BALSLEV.H. , LUTEY N, J., OLLGAARD,B., HOL M-NIEL SEN , L.B.1987 Composition and structure of Adjacent unflooded and floodplain in A mazonian Ecuador. Opera Botánica, 92:37 -57

ENCARNACION, F. 1985 Introducción a la flora y vegetación de la A mazonia peruana estadio actual de los estudios, medio natural y ensayo de una clave de determinación de las formaciones vegetales en la llanura amazónica. Candollea, 40: $237-252$ 
GONZALES RIVADENEYRA, M. 1971 Estudio sobre la densidad de poblaciones de aguaje (M auritia sp. ) en Tingo M aría - Perú. Revista Forestal Del Perú, 5:41 -53

GOTTSBERGER, G. 1978: Seed dispersal by fish in the inundated regions of Huamitá, A mazonia, Biotrópica, 10: 170 - 183

GOULDLNG, M. 1980 the fishes and the forest: Exploration in A mazonian natural history. U niversity of California Press, L os A ngeles.

GRANVILLE，J. J. de 1976 Notes guyanaises: quelques forest sur le Gran mini. Cha. ORSTOM, ser. BioL XI: $23-34$

KAHN, F 1988 iicology of economicaliy important palms in Peruvian Amazonia. A dvances in E conomic Botany, 6: $46-53$

KAHN CASTRO, A. de, 1985 The palm commurdty in a forest of central A mazonia, Brazil. I3iotrópica, 17: 210 - 216

KAHN MEJIA, K., 1991 The palm communities of two terra firme forests in Peruvian A mazonia. Principes, 35 (1): 22-26

KAHN MEJIA, K.; CASTRO A. de 1988 Species richness and density of palms in terra firme forests of A mazonia. Biotrópica, 20

LOPEZ PARODI, J., FREITAS, D. 1990: Geographical aspects of Forested Wetlands in the lower U cayali (Peruvian Amazonía) Forest Ecology and Management 33/34:

MEJIA, K., 1988: Palm ethnobotany in eleven Mestizo villages in thePeru.vianA inaZ Ofl. A dvances in E conomic Botany, 6: 130 - 136

OLDEMAN,R.A.A., 1974 L'architecture de la forét guyanaise. M ém. 73 ORSTOM, Paris

ONERN 1975 Inventario, evaluación e integración de los recursos naturales de la zona de Iquitos, $\mathrm{N}$ auta, Requena y Colonia A ngamos. ONERN, Lima

PRANCE, G.T., 1979 N otes on the vegetation of Amazonía III. The terminology of A mazonian forest types subject to inundation. Brittonia, 31: $26-38$

SALAZAR, A., ROESSL, J., 1977 Estudio de la potencialidad industrial del aguaje. Proyecto ITINTEC No. 3102 UNAIIA, Lima 
SALO. J., KALLIOLA, R., HAKKINEN, IL, MAKINEN, Y., MEMELA, P., PUHAKKA, M., COLEY, P.D. 1986 River dynamics and the diversity of A mazon lowland forest. Nature, 322: 254 - 258

SPRUCE, R., 1871 Palmae A mazonicae. Journ. Linn. Soc. Bot. , 11: 65- 183

TAKEUCHI, M., 1962 The structure of the A mazonian vegetation VI. Igapó J. Fac. Sci. Univ. Tokyo, Sect. 3, Bot. 8: 297 - 304 